\title{
Empleo de aplicaciones tecnológicas en el tratamiento de temas de Probabilidad y Estadística. Dificultades presentadas por los estudiantes en la formulación de planteamientos correctos
}

\author{
Use of technological applications in the treatment of Probability and \\ Statistics subjects. Difficulties presented by students in formulating correct \\ approaches
}

Uso de aplicações tecnológicas no tratamento de problemas de Probabilidade e Estatística. Dificuldades apresentadas pelos alunos na formulação de abordagens corretas

Elena Fabiola Ruiz Ledesma Instituto Politécnico Nacional, Escuela Superior de Cómputo, México

ORCID ID: 0000-0002-1513-8243

\section{Resumen}

En la presente investigación se aborda la problemática de la dificultad que presentan los estudiantes en el planteamiento de soluciones adecuadas a problemas que comúnmente se emplean en la materia de Probabilidad y Estadística en el nivel superior. Se encontró que tanto las diversas temáticas abordadas en los problemas de probabilidad, las cuales no les son familiares a los estudiantes, además del lenguaje que es empleado en los textos, les causa conflicto y una inadecuada interpretación de los problemas, lo que los conduce a proponer un planteamiento erróneo en sus soluciones.

El marco conceptual que soporta este estudio con respecto a la relación que existe entre el lenguaje y las matemáticas proviene de las ideas de Chomsky que subrayan la importancia de esclarecer aspectos semánticos en los problemas de matemáticas, así como las de Duval, quien pone énfasis en las representaciones y sus implicaciones para el estudio 
de los procesos de enseñanza y aprendizaje de las matemáticas. Con respecto a su desarrollo, este estudio tiene un enfoque social constructivista debido a los contextos que abordan los problemas con los que se trabajan.

Se empleó una metodología experimental al trabajar con un grupo de 32 estudiantes quienes cursaban la materia de Probabilidad y Estadística en su tercer semestre de la carrea de Ingeniería en Sistemas Computacionales. Durante el proceso de enseñanza y aprendizaje de dicha materia, se propuso el empleo de diferentes plataformas y aplicaciones tecnológicas para tener un acercamiento diferente al usual.

Tanto los estudiantes como los docentes que participaron en el estudio fueron notificados del trabajo que se realizaría, respetando su decisión en la participación de este.

Primero, se aplicó un cuestionario para conocer su percepción en relación con el uso de la tecnología; después y durante un mes, se trabajó con el grupo con distintas aplicaciones tecnológicas; y finalmente, se aplicó un cuestionario de evaluación.

Los resultados indicaron que uno de los recursos o estrategias que ayudan en la comprensión de los problemas es el uso de material videográfico que represente las situaciones planteadas, permitiendo al estudiante visualizar diversos aspectos de los problemas y no solo trabajar en el terreno algebraico y con el uso exclusivo de fórmulas. Además, la formulación de varias preguntas condujo al estudiante tanto a la determinación de las variables involucradas, como a aclarar lo que se está solicitando medir, evaluar o encontrar en un problema.

Palabras clave: aplicaciones tecnológicas, distribuciones de probabilidad, educación, nivel superior, probabilidad condicional. 


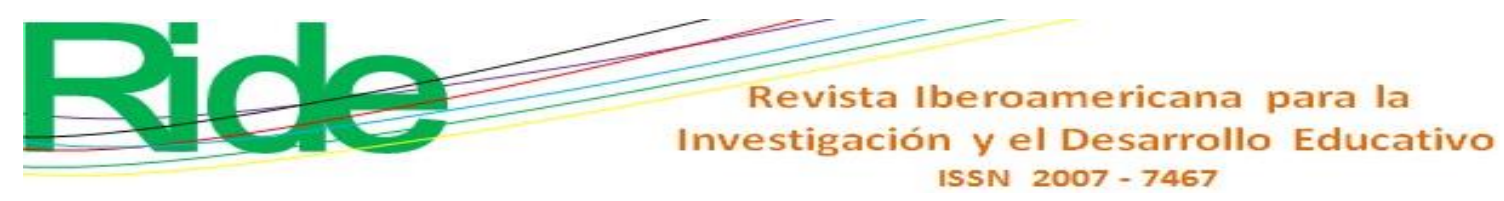

\section{Abstract}

The present research is focused in the difficulty that the students show in the approach of adequate solutions to problems that are commonly used in the field of Probability and Statistics at the College. It was found that both the various issues addressed in the problems of probability, the cells are not familiar to students, in addition to the language that is commonly used in the texts, causes conflict to propose an appropriate approach in their solutions.

The conceptual framework that supports the study in relation to the link between language and mathematics are the ideas of Chomsky on the importance of clarifying semantic aspects in the problems of mathematics and those of Duval who emphasizes the representations and their implications for the study of the teaching and learning processes of mathematics. In relation to the development of the study, it has a constructivist social approach due to the contexts that address the problems with which they work.

Both the students and the teachers who participated in the study were notified of the work that would be done, respecting their decision in the participation of the same.

The experimental methodology was used when working with a group of 32 students who studied the subject of Probability and Statistics in the third semester of the field of Engineering in Computer Systems. First a diagnostic questionnaire was applied to know its perception in relation to the use of technology, after and during a month was worked with the group different applications and finally an evaluation questionnaire was applied. The results indicated that some resources or strategies that help in the understanding of the problems, is the use of videographic material that represents the presented situations, allowing the student to visualize various aspects of the problems and not only work in the algebraic terrain and with the exclusive use of formulas. In addition the formulation of several questions that lead the student both to the determination of the variables involved, to clarify what is being asked to measure, evaluate or find in a problem.

Keywords: technological applications, probability distributions, education, college, conditional probability. 


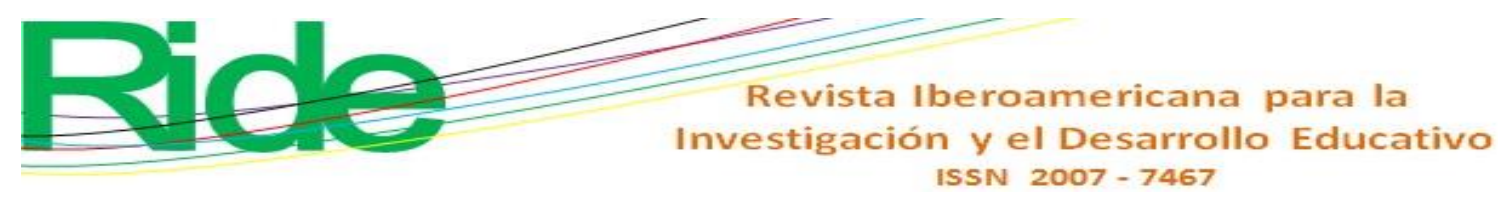

\section{Resumo}

Na presente investigação, aborda-se o problema da dificuldade que os alunos apresentam na abordagem de soluções adequadas aos problemas que são comumente usados na questão de Probabilidade e Estatística no nível superior. Verificou-se que ambas as várias questões abordadas nos problemas de probabilidade, que não são familiares para os alunos, além da linguagem que é usada nos textos, provocam conflitos e uma interpretação inadequada dos problemas, o que os leva a propor uma abordagem errada em suas soluções.

$\mathrm{O}$ quadro conceitual que apóia esse estudo em relação à relação entre linguagem e matemática vem das idéias de Chomsky que enfatizam a importância de esclarecer aspectos semânticos em problemas de matemática, bem como os de Duval, que enfatiza as representações e suas implicações para o estudo dos processos de ensino e aprendizagem de matemática. Com respeito ao seu desenvolvimento, este estudo tem uma abordagem social construtivista devido aos contextos que abordam os problemas com os quais eles trabalham. Uma metodologia experimental foi utilizada ao trabalhar com um grupo de 32 alunos que estudaram o tema de Probabilidade e Estatística em seu terceiro semestre do curso de Engenharia de Sistemas Informáticos. Durante o processo de ensino e aprendizagem deste assunto, o uso de diferentes plataformas e aplicações tecnológicas foi proposto para ter uma abordagem diferente da usual.

Estudantes e professores que participaram do estudo foram notificados do trabalho que seria feito, respeitando sua decisão na participação deste.

Em primeiro lugar, foi aplicado um questionário para conhecer sua percepção em relação ao uso da tecnologia; Depois e durante um mês, trabalhamos com o grupo com diferentes aplicações tecnológicas; e, finalmente, foi aplicado um questionário de avaliação.

Os resultados indicaram que um dos recursos ou estratégias que ajudam na compreensão dos problemas é o uso de material videográfico que representa as situações colocadas, permitindo que o aluno visualize vários aspectos dos problemas e não apenas trabalhe no campo algébrico e com o uso exclusivo de fórmulas. Além disso, a formulação de várias questões levou o aluno à determinação das variáveis envolvidas e a esclarecer o que está sendo pedido para medir, avaliar ou encontrar em um problema. 
Palavras-chave: aplicações tecnológicas, distribuições de probabilidade, educação, nível superior, probabilidade condicional.

Fecha Recepción: Abril $2017 \quad$ Fecha Aceptación: Diciembre 2017

\section{Introducción}

En la materia de Probabilidad y Estadística a nivel superior el planteamiento de los problemas o ejercicios suele ser sobre temas muy variados y algunos de estos son muy extensos (Batanero y Sánchez, 2005). Debido a ello, la premisa que se maneja en la presente investigación es que el estudiante comete errores en la interpretación o en la comprensión del problema, lo que lo conduce a un resultado erróneo. Asimismo, presupone que con la ayuda de material gráfico, animaciones digitales, así como programas computacionales, el estudiante puede llegar a tener un acercamiento que lo lleve a interpretar de forma correcta el problema y alcanzar su solución.

Cai (2003, p. 245) y Lester y Charles (2003, p. 129) comentan que para ayudar a los estudiantes a resolver problemas exitosamente es necesario concientizarlos de que las habilidades de resolución a menudo se desarrollan lentamente, requiriendo atención sostenida a largo plazo y con ello hacerles ver a que resolver problemas es una parte integral del programa de matemáticas. Además, los maestros deben desarrollar una cultura en el aula

que ayude a que la resolución de problemas sea una actividad regular, consistente y práctica. Los estudiantes también deben comprender la importancia de participar regularmente en ejercicios desafiantes.

Para resolver un problema se requiere emprender un proceso que parte de la interpretación del texto matemático; asimismo, hay varios momentos iterativos en donde se realizan pruebas, se ordenan datos, se combinan estos, se emplean conceptos matemáticos y se verifica la información, así hasta llegar a determinar la respuesta (Lesh y Zawojewski, 2007). Polya (1985, pp. 5-6), señaló que todo proceso de solución a un problema requiere 

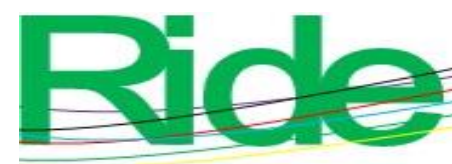

Revista Iberoamericana para la Investigación y el Desarrollo Educativo ISSN $2007-7467$

atravesar cuatro etapas: la comprensión del problema, diseñar un plan, ejecutarlo y verificar lo obtenido. Más tarde, Shoenfeld (1992, p. 339) manifestó que cada etapa de las establecidas por Polya debía ser a su vez dividida, con la finalidad de ser más minucioso al emplear diferentes estrategias (heurísticas y metaheurísticas) que condujeran hasta la solución del problema.

Es por ello que, en la presente investigación, se propuso el uso de elementos, material gráfico y herramientas computacionales a lo largo del proceso implicado en la resolución de los problemas de la asignatura de Probabilidad y Estadística. Esta propuesta se implementó en un grupo de 32 estudiantes pertenecientes a la Escuela Superior de Cómputo del Instituto Politécnico Nacional [ESCOM-IPN] (2009), tomando como referencia los libros de texto señalados en el programa de estudio, con el objetivo de que el estudiante lograra una mejora continua en la comprensión lectora de problemas relacionados con la unidad de aprendizaje en cuestión.

\section{Estado del arte}

La investigación científica acerca de la relación de la matemática y el lenguaje ha mostrado de forma general que es intrínseca tanto en su aprendizaje como en su aplicación por parte de los estudiantes, además de ser sustancial y llevar varias décadas en el ámbito pedagógico.

Monroe (1996) propone la realización de un análisis entre la relación que guarda el lenguaje y la matemática para establecer nuevas estrategias de enseñanza. Para ello, el enfoque se da en la aplicación de las matemáticas a la vida cotidiana, partiendo de la premisa de que el estudiante adquiere conocimientos sólidos y tiene además una percepción favorable de tal ciencia.

Diversos estudios han identificado al lenguaje como uno de los procesos más importantes para aprender matemáticas y para resolver problemas, entre ellos está el realizado por Filio (2005), quien hace referencia al estudio teórico de Chomsky, manifestando que antes de presentar un contenido matemático se requiere definir exactamente cuál es la función del lenguaje en la matemática y cómo influye en su desarrollo como ciencia; a la vez que coloca al lenguaje y a las matemáticas como intermediarios para 
el desarrollo del pensamiento. Otra parte importante de la investigación realizada por Filio tiene que ver con el análisis del lenguaje de algunas áreas de las matemáticas, así como el empleo de un lenguaje más comprensible por su forma, alejado del significado matemático.

Por su parte, García (2014), quien trabaja con estudiantes universitarios, considera a la relación entre el lenguaje y las matemáticas como un problema de comunicación, aunque, aclara, no es único; manifiesta que es la vía principal entre el docente y el alumno, teniendo el primer sujeto cuestionamientos sobre los problemas que sospecha se presentan al impartir la clase. García (Ibidem) señala que los errores que cometen los estudiantes en la resolución de problemas de matemáticas no solo se reducen a respuestas incorrectas, sino que además están presentes muchos componentes, entre ellos la interpretación incorrecta del lenguaje. Al respecto, Rico (1995) comenta que se produce un error si se traduce de forma no correcta una situación matemática de un lenguaje de símbolos a otro diferente. Comparte esta aseveración Guevara (2007), al señalar que en el acto de leer un texto no solo se está descifrando un código, sino también se está dando sentido y significado al mismo.

García (2014) agrega que de forma general los alumnos consideran a la matemática como memorizar una serie de fórmulas y realizar varias operaciones numéricas. Asimismo, menciona que esta forma de pensar afecta a la comunicación matemática, ya que se produce una brecha entre el lenguaje del estudiante y el lenguaje organizado de las matemáticas.

Por su parte, Duval (2004) señala que la comprensión de textos en matemáticas es considerada como una actividad cognitiva, por lo que es necesario emplear distintos registros de representación, como el algebraico, el numérico y el gráfico, que son diferentes al lenguaje común.

D’Amore (2006) comenta que para explicar algunos conceptos los profesores de forma regular emplean un "lenguaje escolar" y que en algunas ocasiones no logran esclarecer la comprensión de la situación planteada, porque cuando se trabaja en matemáticas se aborda una sintaxis particular.

Se puede decir que cada una de las matemáticas emplea una forma de lenguaje y símbolos particulares y que la mayoría de las veces se diferencian dependiendo del contexto o de su utilidad. 
Respecto a la probabilidad y su aprendizaje, Batanero y Sánchez (2005) hacen referencia a algunos objetivos clave para los estudiantes de esta rama del saber, a los cuales, además, consideran valiosos en un ámbito profesional:

- Tener clara la idea de variabilidad de datos y poder resumirla aplicando la teoría de la probabilidad.

- Distribuciones normales como modelos útiles aun cuando sean rara vez perfectos.

- Realizar inferencias válidas a partir de mediciones previas.

- La correlación entre dos variables no implica causa y efecto.

- La estadística puede probar y llevar a conclusiones cercanas a la realidad, y, aunque puedan sugerir hechos, esto debe ser aceptado como irrefutable.

Algunos de los principales problemas para un aprendizaje efectivo de la asignatura de Probabilidad y Estadística se deben a problemas de percepción (Batanero y Sánchez, Ibidem), los cuales se relacionan a conceptos como:

- Representatividad: a partir de una pequeña muestra (o ejemplos) se tiende a generalizar para una muestra mucho más grande, un error de muchos estudiantes y personas en general.

- Falacia de conjunción: creer que dos eventos son más probables que uno solo (sin ser dependientes), aun cuando sea falso en la mayoría de casos.

Así, Batanero y Sánchez (Ibidem) basándose en artículos de la misma temática, encuentran que muchos de estos tienen en común la conclusión de que hay un razonamiento inapropiado acerca de algunas ideas sobre estadísticas y probabilísticas extendido, persistente y similar en todas las edades.

Por otro lado, Lesh y Zawojewski (2007) señalan que en la resolución de problemas no se debe de perder de vista la interpretación matemática de las situaciones que son propuestas, porque muchas veces los estudiantes no usan un pensamiento matemático, sino que involucran aspectos de su experiencia cotidiana.

La investigación desarrollada y presentada en este documento también se basa en el enfoque social constructivista del aprendizaje de las ciencias (Sierpinska y Lerman, 1996; Prados y Cubero, 2005; Pons y Serrano, 2011), en donde se busca que el estudiante construya 

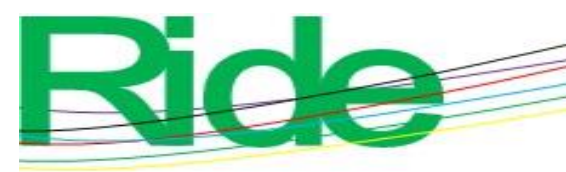

Revista Iberoamericana para la

Investigación y el Desarrollo Educativo

ISSN $2007-7467$

los significados apoyándose en sus conocimientos previos y mediante una actividad o un problema contextualizado, así como que pueda trabajar de forma colectiva.

Justamente los problemas que se presentan en la materia de Probabilidad y Estadística corresponden a muy variados contextos, de tal forma que la propuesta del profesor sobre el análisis que se realice en clase puede ser de forma colectiva.

\section{Problemática de estudio}

Existen varios factores por los cuales los estudiantes no logran obtener un buen rendimiento en la unidad de aprendizaje de Probabilidad y Estadística en la ESCOM-IPN. En el estado del arte se hace referencia a la importancia que guarda la relación entre el lenguaje y las matemáticas y que debido a la inapropiada comprensión de los textos se cae en errores de índole matemático. El factor que en esta investigación se consideró fue referente a las temáticas que abordan los problemas, quedando el planteamiento del problema de la siguiente manera: la gran variedad y cantidad de temáticas, no afines al estudiante y que se abordan en los problemas de la unidad de aprendizaje de Probabilidad y Estadística de la mayoría de los libros de texto (Walpole, Myers y Ye, 2012; Meyer, 1999), constituye una de las muchas causas que lo conducen, al estudiante, a cometer errores y a no enfrentar con éxito los problemas propuestos en clase.

Por todo lo anterior, en la presente investigación se tiene como propósito ayudar a los alumnos y docentes en la compresión e interpretación de textos matemáticos, específicamente textos de Probabilidad y Estadística. Y así contribuir a que los estudiantes resuelvan de mejor manera los ejercicios de dicha asignatura y vincular el contenido de estas situaciones al mundo social en el que se encuentran, ya que la educación en el aula es por naturaleza de tipo social, es decir, su función es que el alumnado tenga un aprendizaje que le permita tanto un desarrollo personal como social (Pons y Serrano, 2011; Punset 2011).

Con el fin de mostrar el resultado del evento que se esté analizando, tener una mejor comprensión del mismo y vaya más allá de un número sin mayor interpretación, se propone usar herramientas didácticas y gráficas como lo son aplicaciones y programas. 


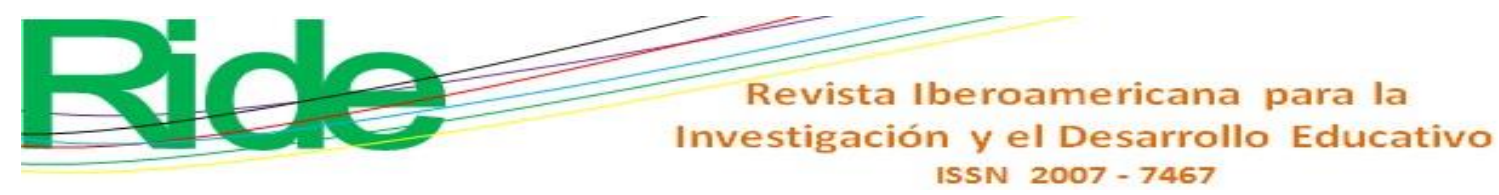

\section{Metodología}

La metodología que se empleó fue de carácter experimental, ya que primeramente se diseñó y aplicó un cuestionario inicial a los estudiantes que estaban cursando durante el turno matutino la asignatura de Probabilidad y Estadística de una de las unidades académicas del ECOM-IPN, correspondiente al ciclo escolar 2017/2, es decir, correspondiente al ciclo de Enero-Junio del 2017 (261 estudiantes). Una vez obtenidas las respuestas, se analizaron y se revisaron las coincidencias que guardan con la literatura consultada.

Paralelamente se hicieron entrevistas a cuatro profesores que se encontraban impartiendo la materia ya mencionada. La muestra de maestros fue seleccionada de forma aleatoria, ya que se les invitó a los siete profesores que atendían la materia en el ciclo escolar especificado anteriormente y solo cuatro docentes aceptaron participar.

Posteriormente se hizo una indagación y se probaron las herramientas computacionales que hay con relación a los temas que abarca el programa curricular de la unidad académica donde se hizo el estudio (ESCOM-IPN, 2009). Se trabajó con una muestra de 32 estudiantes que integraban uno de los 8 grupos que se tienen en la ESCOM-IPN, en donde estos se conforman de manera aleatoria. Los estudiantes cursaban la materia de Probabilidad en el tercer semestre de la carrera de Ingeniería en Sistemas Computacionales: jóvenes de entre 20 y 23 años. Estos alumnos ya habían llevado cursos de Cálculo, Matemáticas Discretas y Ecuaciones Diferenciales, lo que implica que contaban con las herramientas que ofrecen estas asignaturas. En estos cursos previos habían resuelto problemas en contextos de la física, así como propios de la matemática (geometría, trigonometría, geometría analítica) y de otras áreas como de electrónica y comunicaciones.

A los estudiantes se les pidió que trabajaran durante un mes con algunas aplicaciones tecnológicas, como software y páginas web (Khan Academy, Educatina, Probability Distributions), las cuales se describen más adelante. Los temas que se abordaron fueron la probabilidad condicional y distribuciones de probabilidad.

Después de su uso, se aplicó un cuestionario de evaluación, pero esta vez sólo participaron los 32 estudiantes que trabajaron con las aplicaciones computacionales. En esta parte el interés se centró en evaluar la utilidad que habían encontrado con el uso de estas. 


\section{Instrumentos metodológicos empleados}

\section{Cuestionario inicial dirigido a los estudiantes}

El empleo de herramientas didácticas y gráficas como lo son programas y aplicaciones ayuda al alumno a plantear lo que tendría que resolver en torno a la probabilidad. Para detectar cuáles son las principales dificultades que se tienen al momento de resolver y entender un problema de esta índole, como ya se mencionó anteriormente, se aplicó un cuestionario inicial a los alumnos de la ESCOM-IPN que cursaban la materia de Probabilidad y Estadística, quienes se encontraban inscritos en el tercer semestre del ciclo escolar EneroJunio de 2017, los cuales fueron un total de 261 alumnos. En la tabla 1, se muestra esta encuesta inicial.

Tabla 1. Preguntas del cuestionario inicial para los estudiantes.

1. ¿Qué tan difícil es para ti la probabilidad?

2. ¿Consideras que el aprendizaje depende más de la enseñanza del profesor que del desempeño del alumno?

3. ¿Cuál crees que sea la principal causa por la que no se logra comprender el curso de Probabilidad y Estadística?

4. ¿Cómo lograrías mejorar la parte de entendimiento del curso?

5. ¿Qué dificultades presentas al resolver un ejercicio?

6. Personalmente, ¿qué método de enseñanza te resulta más eficiente para entender la probabilidad y estadística?
7. ¿Qué método utilizarías para poder resolver los ejercicios de esta asignatura?

8. ¿Consideras que sería importante ocupar una parte del curso para analizar la interpretación de los problemas? Sí / No ¿Por qué?

9. ¿Crees que la probabilidad está implícita en hechos de la vida cotidiana? Sí / No ¿Por qué?

10. ¿Opinas que ocupar material didáctico y gráfico ayude a entender mejor el planteamiento del problema?

Fuente: Elaboración propia. 


\section{Forma de trabajo con las aplicaciones computacionales}

Para la implementación de herramientas computacionales como auxiliares en la resolución de problemas, la clase se dividió en cuatro etapas:

1. Inicio: el profesor proponía un problema del tema de probabilidad condicional o de alguna distribución de probabilidad, ya fuera discreta o continua (binomial, exponencial, geométrica o normal). Se auxiliaba de la plataforma Khan Academy para mostrar un video relacionado con el problema planteado.

2. Desarrollo: el profesor formulaba una serie de preguntas respecto al problema presentado. Una vez que el estudiante reconocía la distribución de probabilidad que podía emplear para dar solución al problema, empleaba el software Statistics Calculator o el software Probability Distributios para realizar los cálculos.

3. Evaluación: en esta parte de la clase el profesor empleaba la plataforma Khan Academy para presentar al estudiante problemas del mismo tema y le daba tiempo a este para trabajar en dicha plataforma. En ella los alumnos podían revisar videos en donde se mostaban procesos de solución, así como se daban consejos para la solución de los problemas. También en la misma plataforma se calificaban los problemas y ejercicios resueltos por los alumnos.

4. Retroalimentación: el profesor invitaba a los estudiantes a que explicaran algunos de los problemas revisados y resolvía las dudas que se tuvieran.

\section{Aplicaciones Computacionales}

Probability Distributions: es una app disponible en Android para las distribuciones de probabilidad, tales como la distribución binomial, de geométrica, de Poisson, de hipergeométrica y la distribución binomial negativa, así como probabilidades de cómputo, determinar los percentiles, el argumento de la función de densidad de probabilidad para la normal (Gaussiana), t, chi-cuadrada, F, exponencial, gamma, beta y distribuciones normales de registro Matthew Bognar (2017).

Esta app fue de mucha ayuda debido a que redujo el tiempo de búsqueda de los datos requeridos. Primero se detectaron las variables de los problemas, con ello se analizaron las posibles distribuciones que podrían usarse $\mathrm{y}$, finalmente, se determinó la que era la más adecuada. Después se introducían los valores en las casillas correspondientes y la aplicación 
arrojaba las respuestas correspondientes. Fue un gran apoyo, puesto que mostraba las gráficas y no solo los resultados numéricos, lo que permitía realizar una interpretación de lo obtenido. En la figura 1 se muesta la imagen de una de las interfaces.

Fgura 1. Interfaz gráfica de Probability Statics.

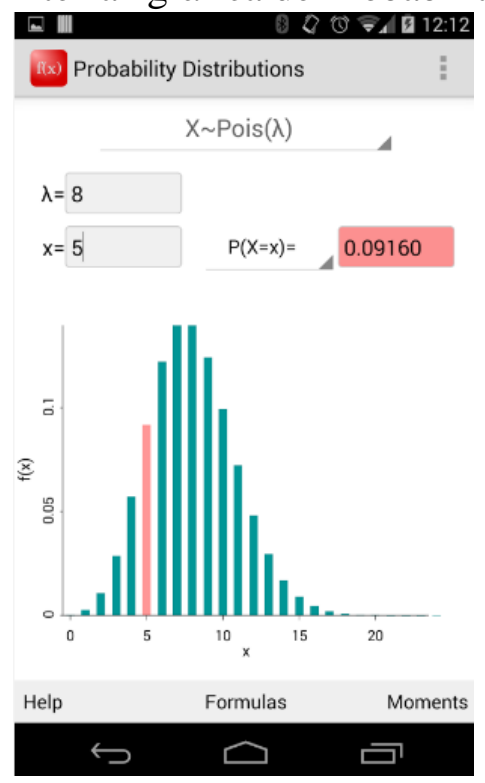

Fuente: Matthew Bognar, 2017.

Statistics Quick Reference: aplicación disponible para Android con versión gratuita o de paga (Statistics Quick Reference, 2017). La aplicación dispone de un pequeño diccionario introductorio que comprende los conceptos y las definiciones básicas para familiarizarse con la terminología de la materia. Cada uno de los temas a explicar está seguido por un ejemplo numérico con el fin de asentar los conocimientos y asegurar la compresión de la definición. (Fig. 2). Esta aplicación aportó ideas para la comprensión de los problemas trabajados.

Educatina: esta página web cuenta con 8 secciones que a su vez cada una tienen varios subtemas, según sea la materia seleccionada. En la sección de Probabilidad y Estadística se tiene un amplio índice de temas. Es una página didáctica que ayuda al estudiante a entender los temas, reforzando el entendimiento con ejercicios. Además, cuenta con la opción de calificar dichos ejercicios y se muestra si la respuesta seleccionada es errónea y, en tal caso, arroja la correcta. 


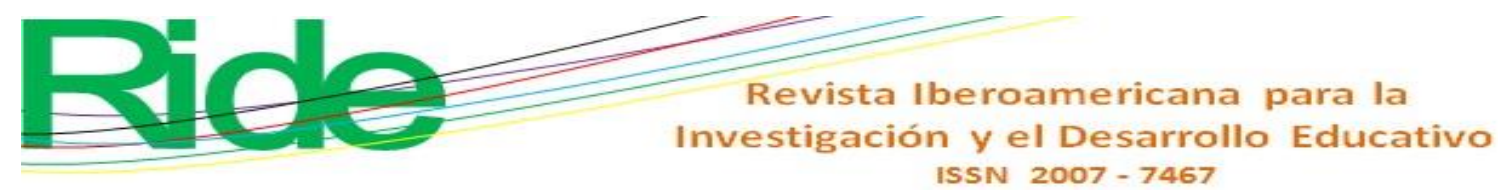

Figura 2. Interfaz gráfica de Quick References.

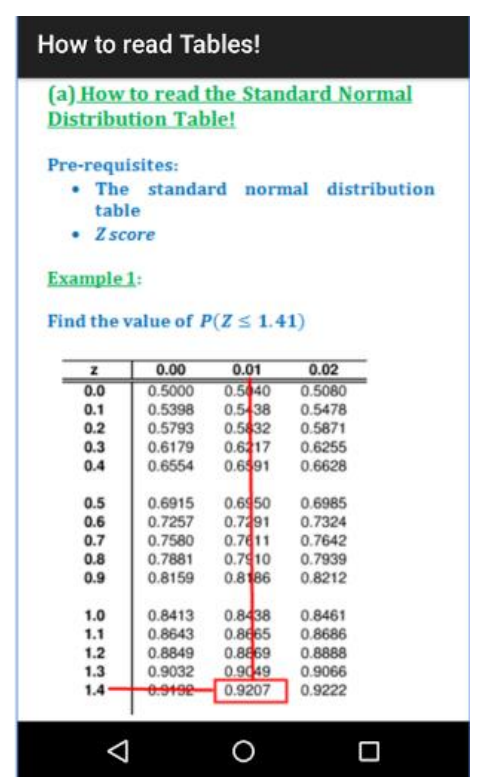

Fuente: Statistics Quick References, 2017.

En este aplicación el estudiante encontró la explicación a varios problemas que se le planteban, porque se usan gráficos para representar lo que se está formulando en el problema, así como se formulan preguntas que ayudan a desmenuzar el ejercicio en partes.

Statistics Calculator: Esta app está disponible en Android y cuenta con 8 distribuciones: Binomial, Normal, Uniforme, Poisson, Exponencial, Gamma, Geométrica, y Joint Distribution. Además de calcular las probabilidades, calcula la covarianza y correlación para la distribución conjunta, incluyendo media, desviación estándar y la varianza para todas las distribuciones (Statistics Calculator, 2017). Esta aplicación se empleó con los estudiantes para calcular los valores de las distribuciones que permitirían resolver los problemas planteados.

Khan Academy: Es una página web donde se encuentran tres secciones, principalmente: alumno, profesor y papás. Aquí se encuentran varios temas de estudio, en específico hablando de probabilidad y estadística. Asimismo, contiene un índice de temas, en donde, por medio de un video, se explican cada uno de ellos. Posteriormente cuenta con una sección de ejercicios relacionados al tema anteriormente visto. Cuenta con la opción de poder calificar el resultado mostrando si este es correcto o no (Khan Academy, 2017). 
Esta plataforma se usó en las clases de la siguiente forma. Primero se presentaba un problema al estudiante y se le mostraba un video el cual explicaba lo que se pedía resolver (esto ayudó mucho al alumnado debido a que, como se ha decrito a lo largo del presente artículo, es el punto más débil que tiene la mayoría de quienes lo integran). Después se mostraban problemas con otras variables y se le pedía al estudiante identificarlas, así como identificar lo que se solicitaba determinar. Para ello se recurrió a la formulación de preguntas relacionadas a distintos aspectos del problema y a la respuesta a cada una de ellas. Finalmente el estudiante resolvía diferentes ejercicios y el mismo sistema lo evaluaba.

En la figura 3 se presenta la imagen capturada de uno de los videos que contiene la plataforma de Khan Academy en donde se presenta un problema y mediante el subrayado de algunas palabras le muestra al estudiante lo que se está pidiendo determinar. Después muestra un diseño de solución y lo sigue paso a paso hasta llegar a la respuesta.

Figura 3. Ejemplo del proceso de solución de un problema.

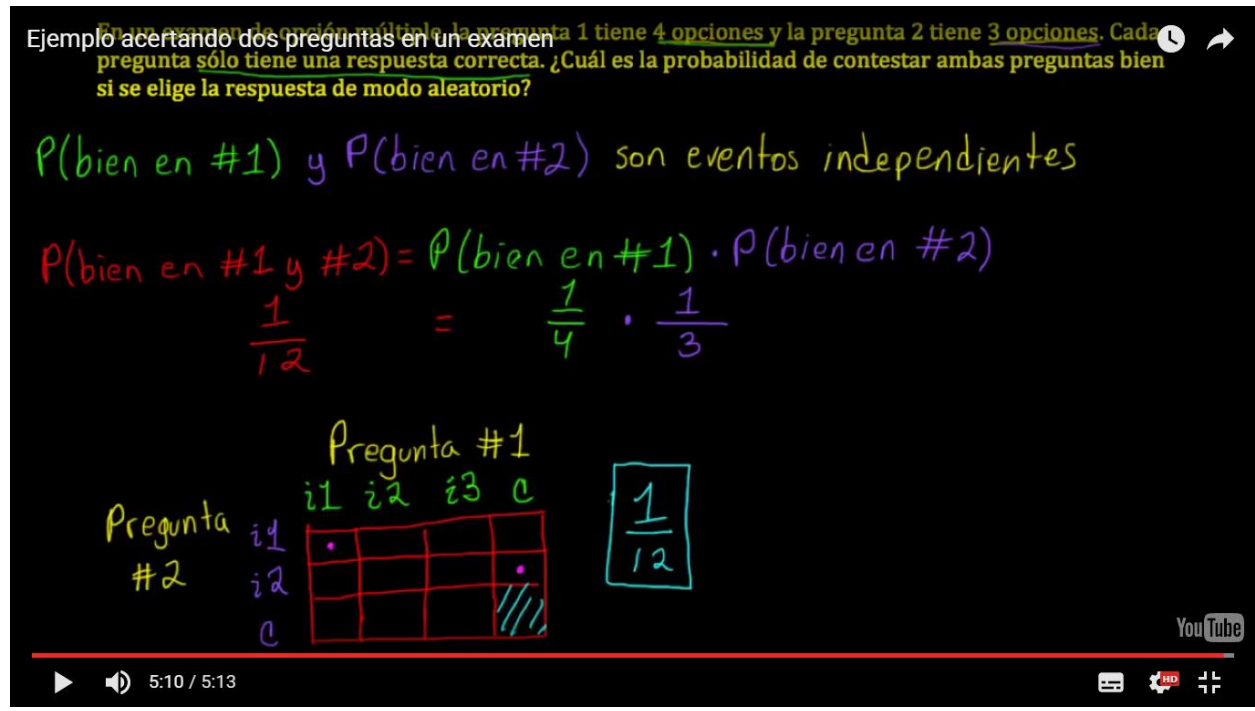

Fuente: Khan Academy, 2017.

En la figura 4 se muestra otro problema y el material gráfico que se empleó para describir paso a paso, tanto la interpretación del texto del problema como de todo el proceso de solución. 

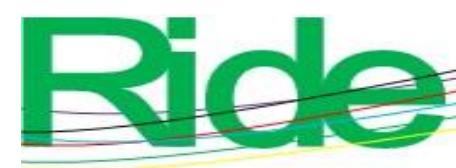

Revista Iberoamericana para la Investigación y el Desarrollo Educativo ISSN $2007-7467$

Figura 4. Ejemplo del proceso de solución de un problema.

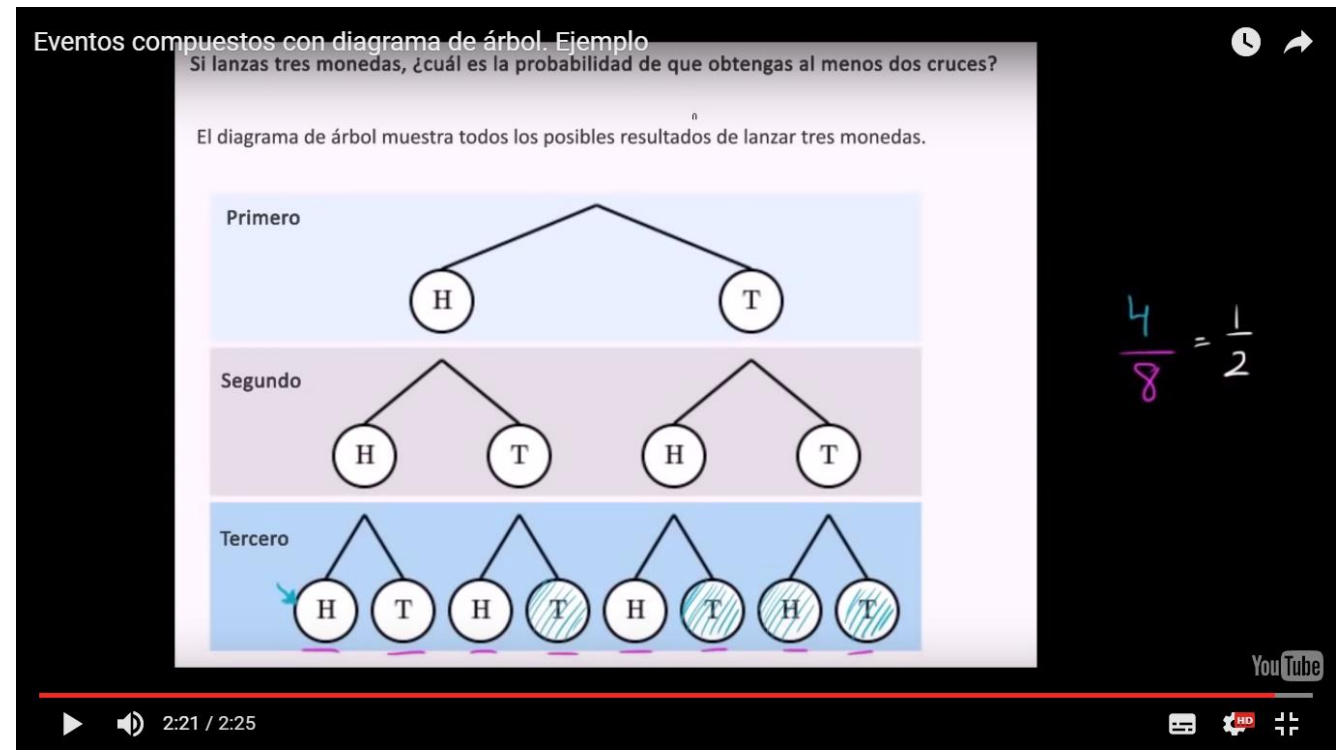

Fuente: Khan Academy, 2017.

\section{Resultados y análisis}

\section{Resultados del cuestionario inicial}

Derivado de la aplicación del cuestionario inicial dirigido a los alumnos, se organizaron las respuestas en categorías para su análisis. Dichas categorías se muestran en la tabla 2. 
Tabla 2. Organización de la información de las respuestas en categorías.

\begin{tabular}{|c|c|c|}
\hline Pregunta & Categorías & Porcentaje \\
\hline $\begin{array}{l}\text { ¿Qué tan difícil es para ti la } \\
\text { probabilidad? }\end{array}$ & $\begin{array}{l}\text { Nivel de dificultad: } \\
\text { Alto } \\
\text { Intermedio } \\
\text { Bajo }\end{array}$ & $\begin{array}{l}70 \% \\
30 \% \\
-\end{array}$ \\
\hline $\begin{array}{l}\text { ¿Consideras que el } \\
\text { aprendizaje depende más de la } \\
\text { enseñanza del profesor que del } \\
\text { desempeño del alumno? }\end{array}$ & $\begin{array}{l}\text { Más del profesor que del alumno } \\
\text { Igual } \\
\text { Más del alumno que del profesor }\end{array}$ & $\begin{array}{l}80 \% \\
10 \% \\
10 \%\end{array}$ \\
\hline $\begin{array}{l}\text { ¿Cuál crees que sea la } \\
\text { principal causa por la que no } \\
\text { se logra comprender el curso } \\
\text { de Probabilidad y Estadística? }\end{array}$ & $\begin{array}{l}\text { Interpretación errónea de los textos de los } \\
\text { problemas. } \\
\text { Planteamiento deficiente }\end{array}$ & $\begin{array}{l}90 \% \\
80 \%\end{array}$ \\
\hline $\begin{array}{l}\text { ¿Cómo lograrías mejorar la } \\
\text { parte de entendimiento del } \\
\text { curso? }\end{array}$ & $\begin{array}{l}\text { Presentar ejemplos prácticos. } \\
\text { Analizar los problemas para formular } \\
\text { planteamientos correctos }\end{array}$ & $\begin{array}{l}70 \% \\
90 \%\end{array}$ \\
\hline $\begin{array}{l}\text { Personalmente, ¿qué método } \\
\text { de enseñanza te resulta más } \\
\text { eficiente para entender la } \\
\text { probabilidad y estadística? }\end{array}$ & $\begin{array}{l}\text { Desglosar en varias preguntas la pregunta } \\
\text { principal del problema. } \\
\text { Utilizar material gráfico que represente el } \\
\text { contenido del problema } \\
\text { Más participación del alumno en clase }\end{array}$ & $\begin{array}{l}48 \% \\
38 \% \\
80 \%\end{array}$ \\
\hline $\begin{array}{l}\text { ¿Qué método utilizarías para } \\
\text { poder resolver los ejercicios } \\
\text { de esta asignatura? }\end{array}$ & $\begin{array}{l}\text { Analizar los problemas para formular } \\
\text { planteamientos correctos. } \\
\text { Desglosar en varias preguntas la pregunta } \\
\text { principal del problema. }\end{array}$ & $\begin{array}{l}90 \% \\
48 \%\end{array}$ \\
\hline $\begin{array}{l}\text { ¿Consideras que sería } \\
\text { importante ocupar una parte } \\
\text { del curso para analizar la } \\
\text { interpretación de los } \\
\text { problemas? Si/No ¿Por qué? }\end{array}$ & $\begin{array}{l}\text { Sí, porque es la base para su solución } \\
\text { No }\end{array}$ & $100 \%$ \\
\hline $\begin{array}{l}\text { ¿Crees que la probabilidad } \\
\text { está implícita en hechos de la } \\
\text { vida cotidiana? Si/No ¿Por } \\
\text { qué? }\end{array}$ & $\begin{array}{l}\text { Sí } \\
\text { En juegos } \\
\text { Aspectos meteorológicos } \\
\text { Toma de decisiones }\end{array}$ & $100 \%$ \\
\hline $\begin{array}{l}\text { ¿Opinas que ocupar material } \\
\text { didáctico y gráfico ayude a } \\
\text { entender mejor el } \\
\text { planteamiento del problema? }\end{array}$ & $\begin{array}{l}\text { Sí } \\
\text { No }\end{array}$ & $100 \%$ \\
\hline
\end{tabular}

Fuente: Elaboración propia 
El 90\% de los estudiantes está de acuerdo con la implementación de la propuesta sobre el uso de tecnología como herramienta en el aprendizaje de esta asignatura, además de que están conscientes que un problema con el que la mayoría se enfrenta es la errónea interpretación y comprensión de ejercicios, cuya redacción les parece larga y confusa. No obstante, también se encontró que los alumnos prefieren diversos métodos de enseñanza, sin que predomine uno en específico.

Ochenta por ciento de los alumnos mencionó que para un buen aprendizaje de la materia se necesita una buena base de enseñanza por parte del profesor. Si bien el alumno tiene la obligación de repasar los temas vistos en clase, el profesor tiene una parte importante en cuanto al desarrollo y comprensión de los problemas. Esto debido a que si el instructor se adjudica todo el proceso de enseñanza y si no logra explicar de manera concreta y correcta cómo es el procedimiento a seguir, el alumno tendrá una idea errónea o incompleta que dará como resultado ejercicios incompletos, datos mal asignados o falta de capacidad de compresión y desarrollo del problema. Pero si el profesor comparte el proceso de enseñanza permitiendo al alumno descubrir, investigar y ser partícipe en la clase, entonces los alumnos tendrán la responsabilidad de buscar opciones y compartirlas con sus compañeros y el propio profesor y así abordar los problemas y los procesos de solución. En esta búsqueda, ambos (profesor y alumnos), tienen la oportunidad de revisar diferentes aplicaciones tecnológicas y plataformas que cuenten con material o recursos que sirvan de apoyo en esta tarea.

En este último punto, los métodos de enseñanza preferidos por los estudiantes fueron los siguientes: el $38 \%$ por ciento de los alumnos concuerda que el mejor método de enseñanza que podrían tener es mediante ejercicios gráficos, debido a que se tiene una visión y mayores magnitudes del problema. Mientras que el 14\% coincide que la manera menos eficiente para entender un problema es resolviendo ejercicios mediante enunciados, debido a que en muchas ocasiones estos vienen redactados de una manera confusa y larga, por lo que, en el proceso de recopilación de datos, el alumno tiende a perder de vista el objetivo general del ejercicio, causando que lea el problema más de una vez para entender el punto a donde se quiere llegar y recopilar la información correcta. 
Por otro lado, el $90 \%$ de los estudiantes encuestados está de acuerdo en que es importante dedicar un tiempo considerable durante el curso a la interpretación y análisis de cada problema, con la finalidad de tener un mejor conocimiento en relación al surgimiento de dichos datos y el significado del resultado que se obtenga, ya que la probabilidad está implícita en hechos de la vida cotidiana, aunque no siempre de forma directa, como lo es en la toma de una decisión para poder ganar un volado, en juegos de mesa o juegos de lotería y en muchos ámbitos más que se desarrollan en el día a día.

Concluyendo, la gran mayoría de los alumnos concuerda que el uso de herramientas gráficas facilitaría la mejora en la comprensión de la materia. También concuerdan en que depende mucho de la manera en cómo el profesor abre el panorama al alumno en cuanto a la resolución de los problemas y su interpretación.

\section{Entrevista a profesores}

Se entrevistó a 4 de los profesores que imparten la materia en la escuela donde se realizó el estudio, se les pidió autorización de ser grabados, después la información proporcionada en las preguntas abiertas de la entrevista fue ordenada mediante el establecimiento de categorías, mismas que fueron empleadas para su análisis. Esta información se presenta en la tabla 3. 
Tabla 3. Preguntas de la entrevista realizada a los profesores y concentración de resultados.

\begin{tabular}{|c|c|c|}
\hline Pregunta & Categorías & Porcentaje \\
\hline $\begin{array}{l}\text { ¿Considera que el curso de } \\
\text { probabilidad y estadística es } \\
\text { complejo? Sí / No ¿Por } \\
\text { qué? }\end{array}$ & $\begin{array}{l}\text { Por la cantidad de temas que tiene. } \\
\text { Por el tipo de problemas a resolver. } \\
\text { Por el poco tiempo con el que se } \\
\text { cuenta para impartir todo el temario. } \\
\text { Por la complejidad matemática de los } \\
\text { temas. }\end{array}$ & $\begin{array}{l}100 \% \\
100 \% \\
100 \% \\
75 \%\end{array}$ \\
\hline $\begin{array}{l}\text { ¿Dónde fallan los alumnos } \\
\text { con más frecuencia al ver } \\
\text { los temas? }\end{array}$ & $\begin{array}{l}\text { En la interpretación de los textos de } \\
\text { los problemas. } \\
\text { En la selección de la distribución } \\
\text { adecuada, es decir, decidir si } \\
\text { corresponde a una distribución } \\
\text { binomial o a una geométrica negativa. } \\
\text { En la selección correcta de la fórmula. } \\
\text { En el planteamiento correcto del } \\
\text { problema. }\end{array}$ & $100 \%$ \\
\hline $\begin{array}{l}\text { ¿Qué método de enseñanza } \\
\text { consideras que es el más } \\
\text { apto para que el alumno } \\
\text { entienda bien los temas? }\end{array}$ & $\begin{array}{l}\text { Resolución de problemas en clase. } \\
\text { Leer más de una vez los problemas. } \\
\text { Determinar las variables del problema } \\
\text { y sus relaciones. }\end{array}$ & $\begin{array}{l}100 \% \\
100 \% \\
75 \%\end{array}$ \\
\hline $\begin{array}{l}\text { ¿Utilizas alguna } \\
\text { herramienta como apoyo } \\
\text { para que el alumno entienda } \\
\text { mejor los temas? Sí / No } \\
\text { ¿Cuál? ¿Por qué? }\end{array}$ & $\begin{array}{l}\text { Calculadora. } \\
\text { Libros de texto. } \\
\text { No usan aplicaciones tecnológicas. }\end{array}$ & $\begin{array}{l}100 \% \\
100 \% \\
100 \%\end{array}$ \\
\hline $\begin{array}{l}\text { ¿Cuál consideras que es el } \\
\text { principal problema por el } \\
\text { cual los alumnos no } \\
\text { comprenden bien los temas? }\end{array}$ & $\begin{array}{l}\text { La falta de comprensión de los textos } \\
\text { de los problemas. } \\
\text { No tienen un buen planteamiento. } \\
\text { No ponen atención en las clases. }\end{array}$ & $\begin{array}{l}100 \% \\
100 \% \\
50 \%\end{array}$ \\
\hline
\end{tabular}

Fuente: Elaboración propia.

Como resultado de las entrevistas realizadas a los profesores, se encontró que la mayor parte considera que el curso de Probabilidad y Estadística tiene un temario extenso para cubrirlo completamente durante un semestre. Asimismo, mencionaron que un problema común en los estudiantes es cometer errores en el planteamiento del problema, debido a una mala interpretación que, de acuerdo con los profesores encuestados, es por la falta de atención durante la clase, además de los muchos temas que deben impartirse, sin olvidar que algunos de los problemas están redactados de manera confusa. 
Los profesores a los que se les aplicó el cuestionario propusieron una serie de medidas para disminuir la incidencia de este problema:

- $\quad$ Leer más de una vez el problema.

- Subrayar palabras claves que pudieran indicar la necesidad de hacer determinado proceso.

- $\quad$ Apoyo de tablas de probabilidad, de aplicaciones que sirvan para corroborar resultados obtenidos y de libros de la unidad de aprendizaje con sus ejercicios.

- Resolver listas de ejercicios de cada tema.

- Asistir a asesorías cuando un tema no esté comprendido completamente.

En los resultados, además, se destaca que el $60 \%$ de los encuestados concuerda en que la unidad de aprendizaje de Probabilidad y Estadística resulta difícil debido a la interpretación errónea de los problemas, puesto que se tienen diferentes lógicas de resolución para un mismo problema, tanto para los alumnos y profesores como para los autores de los ejercicios.

Se propusieron estas aplicaciones con el fin de ayudar al estudiante y profesor en el proceso de plantear y resolver problemas de los diferentes temas de la signatura y plantel educativo ya mencionados.

Después de trabajar con las aplicaciones antes mostradas se encontró lo siguiente:

- $\quad$ Permitió a los estudiantes visualizar las situaciones que se mostraban en forma de problemas, contribuyendo a que mejoraran en el planteamiento de lo que debían resolver.

- Hubo una mejora en cuanto al tiempo empleado en la resolución de los problemas que se resolvieron.

- $\quad$ Se pudo ratificar las respuestas obtenidas de los ejercicios que previamente se tuvieron que realizar en el pizarrón, individualmente o en grupos.

Se puede concluir que, al usar estas aplicaciones o sitios web, el rendimiento del alumno fue mayor y el índice de error que se tiene en cuanto a la resolución de los problemas bajó notablemente. Igualmente importante es que parte de los alumnos mencionaron que les fue más fácil comprender los temas y, en caso de tener algún error, notar la falla que se tuvo. 


\section{Cuestionario de evaluación del uso de las aplicaciones}

En la tabla 4 se muestran las preguntas que se realizaron a los 32 estudiantes que trabajaron durante un mes con las aplicaciones antes descritas.

Tabla 4. Preguntas del cuestionario de evaluación para los estudiantes.

Después de haber trabajado con diferentes aplicaciones y páginas web en tu curso de Probabilidad y Estadística te pedimos responder las siguientes preguntas:

1. ¿En qué consideras que te ayudaron las aplicaciones?

2. ¿Recomendarías el uso de estas aplicaciones en el curso de Probabilidad? Sí / No ¿Por qué?

3. ¿Qué otra técnica propones para ayudar a la comprensión de los textos de los problemas de probabilidad?

4. Resuelve los problemas que aparecen en la hoja anexa titulada como "Examen de la unidad 3". Puedes auxiliarte de una calculadora para hacerlo.

\section{Fuente: Elaboración propia.}

Respecto al cuestionario de evaluación aplicado a los 32 alumnos sobre el desarrollo de técnicas para un mejor entendimiento de problemas matemáticos (específicamente de la asignatura de Probabilidad y Estadística), se encontró que el $85 \%$ de los estudiantes considera que el empleo de estas herramientas reduce el tiempo que se invierte en la resolución de los problemas con relación a cuando solo trabajan con un cuaderno y una calculadora básica. Setenta y seis por ciento señaló que le ayudó a tener claridad en lo que pide el problema y el 90\% comentó que las aplicaciones le permitieron revisar cómo ocurren los eventos de los problemas planteados. Además, un $96 \%$ consideró que si los ejercicios se relacionaran con el área de estudio del alumno tendrían una mejor comprensión e interpretación de ellos. Ochenta y cuatro por ciento consideró que una reducción del número de ejercicios tendría un impacto positivo en la comprensión de los temas que estos abordan, debido a que mencionaron que sus profesores les dejan una lista de muchos problemas y no les alcanza el tiempo para analizar lo que les están pidiendo en el problema, por ello los resuelven usando fórmulas, pero sin mucho razonamiento. 
Asimismo, la totalidad de la muestra de estudiantes recomendó el uso de las aplicaciones porque pueden trabajar con ellas en su celular en cualquier lugar. Setenta y seis por ciento está de acuerdo con el hecho de que, si se usan nuevas tecnologías en la enseñanza de matemáticas (particularmente en el curso de Probabilidad y Estadística), los estudiantes se involucrarían más en los temas. De manera similar a la pregunta del uso de nuevas tecnologías, el 90\% encontró el desarrollo de actividades recreativas para resolución de problemas de Probabilidad y Estadística como una opción para un mejor aprendizaje.

Por su parte, el 56\% se interesó en realizar lecturas relacionadas a matemáticas que permitan el desarrollo del pensamiento crítico, siendo esta otra posible propuesta distinta a la expuesta en este artículo, lo cual se relaciona directamente con el hecho de que el $84 \%$ considera que una reducción del número de ejercicios tendría un impacto positivo en la comprensión de los temas que estos abordan (a causa de que hay una dificultad común entre los alumnos: la interpretación errónea de los enunciados, siendo algunos de estos confusos).

En relación con la forma de cómo se evaluó el trabajo realizado en el aula con las aplicaciones, se aplicó un examen formado por cinco problemas, en donde los alumnos emplearon las aplicaciones para resolverlo. Este examen valió diez puntos y se muestra en la tabla 4. Se evaluó el procedimiento empleado por el estudiante, las estrategias con las que trabajaron y la interpretación de los resultados obtenidos. La escala que se manejó fue de cero a 10, siendo calificaciones aprobatorias las correspondientes al intervalo de seis a 10 y reprobatorias las restantes.

En el anexo 1, se presentan los problemas que resolvieron los estudiantes, los cuales fueron tomados de uno de los libros de texto que es señalado en el programa de estudios de la materia de Probabilidad y Estadística (Walpole, Myers y Ye, 2012; Meyer, 1999).

Para la revisión de las respuestas dadas por los estudiantes se hizo una clasificación en cuatro aspectos. Primero se deseó saber si habían distinguido el tipo de variable que estaba en juego en cada problema, es decir, si era discreta o continúa. Después se revisó el tipo de distribución de probabilidad a la que corresponden dichas variables, de acuerdo con su comportamiento. Enseguida se identificó si el estudiante había empleado alguna de las aplicaciones trabajadas en clase, para ello se le pidió que escribiera en la hoja del cuestionario la o las aplicaciones empleadas. Finalmente, se contabilizaron los puntos obtenidos en cada 
problema. Cada uno tenía dos incisos a resolver, por lo que cada inciso equivalía a un punto. En total el cuestionario equivalía a 10 puntos. En la tabla 5 se muestra el concentrado de la información expresada en los cuatro aspectos señalados.

Tabla 5. Clasificación de la información obtenida de las respuestas dadas por los estudiantes al cuestionario final.

\begin{tabular}{|l|l|l|l|l|l|l|}
\hline $\begin{array}{l}\text { Núm. de } \\
\text { problema }\end{array}$ & $\begin{array}{l}\text { Identificó } \\
\text { la variable } \\
\text { (discreta o } \\
\text { continua) }\end{array}$ & $\begin{array}{l}\text { Identificó la } \\
\text { distribución } \\
\text { de } \\
\text { probabilidad }\end{array}$ & $\begin{array}{l}\text { Usó alguna } \\
\text { app oducativa } \\
\text { plataforma } \\
\text { edún }\end{array}$ & $\begin{array}{l}\text { Operaciones } \\
\text { correctas }\end{array}$ & $\begin{array}{l}\text { Respuestas } \\
\text { correctas }\end{array}$ \\
\hline 1 & Sí (29/32) & Sí (28/32) & Sí (18/32) & Sí $(29 / 32)$ & $29 / 32$ \\
\hline 2 & Sí (27/32) & Sí (27/32) & Sí (19/32) & Sí $(27 / 32)$ & $27 / 32$ \\
\hline 3 & Sí (27/32) & Sí (27/32) & Sí (19/32) & Sí (27/32) & $27 / 32$ \\
\hline 4 & Sí (21/32) & Sí (24/32) & Sí (19/32) & Sí (19/32) & $19 / 32$ \\
\hline 5 & Sí (24/32) & Sí (23/32) & Sí (20/32) & Sí (20/32) & $20 / 32$ \\
\hline
\end{tabular}

Fuente: Elaboración propia.

Veintisiete de 32 estudiantes $(84.4 \%)$ obtuvo una calificación aprobatoria y un estudiante no se presentó al examen.

El promedio del grupo fue de 7.3 con una desviación estándar de 2.6 y el $68.7 \%$ de las calificaciones se encuentran dentro de una desviación estándar con respecto a la media, lo que corresponde a lo señalado por la regla empírica (a una desviación estándar a la derecha y a una a la izquierda se encuentra contenido al menos $68 \%$ de los datos de la muestra).

Los resultados obtenidos con el grupo implican que fueron de gran ayuda las aplicaciones que emplearon en las clases y fuera de ellas, ya que los estudiantes lograron realizar interpretaciones correctas de los textos de los problemas y pudieron resolver adecuadamente las situaciones planteadas. 


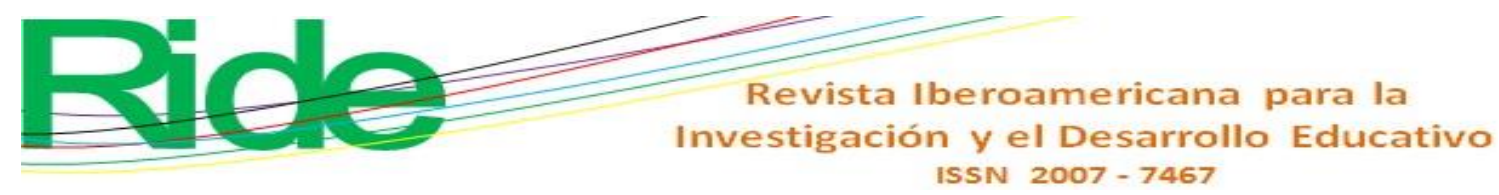

\section{Conclusiones}

La premisa planteada al inicio de la investigación con relación a la dificultad que tienen los alumnos al momento de aprender Probabilidad y Estadística radica en la poca comprensión e interpretación de los problemas planteados en clase. Se trató de una premisa corroborada, aunque se encontraron otros factores. Se encontró que son varios los puntos débiles que propician las dificultades para aprender los temas de esta materia, ya sea por la estructura del programa de la asignatura (muchos temas en poco tiempo) o por el tipo de enseñanza que predomina en los profesores que la imparten (muy recostado en el conductismo), así como en la forma en la que están redactados los problemas que son extraídos de los libros de texto, los cuales están formulados mediante textos amplios y muchas veces rebuscados. Otro aspecto es que la mayoría de los alumnos no consideran una utilidad práctica en los conocimientos abordados de la materia.

Con la información recopilada, por parte de las encuestas y los cuestionarios aplicados a los estudiantes y la entrevista realizada a los profesores, se decidió emplear una estrategia que incorporaba material gráfico, empleo de sitios web y programas de cómputo para poder analizar los contextos de los diferentes problemas que se trabajaban en clase. El trabajo que se desarrolló coincide con lo señalado por Sierpinska y Lerman (1996), respecto a un aprendizaje de tipo constructivista social.

Para que los estudiantes puedan comprender los diferentes contextos que se abordan en los problemas de probabilidad es conveniente emplear material videográfico como el empleado en los sitios web y las aplicaciones, y así el estudiante tenga un acercamiento más eficaz en su proceso de solución.

Por otra parte, el uso de aplicaciones facilitó la comprensión de los diferentes problemas que se resolvieron, permitiendo al estudiante visualizar las situaciones planteadas y no solo trabajar en el terreno algebraico y con el uso exclusivo de fórmulas.

Se concluye que, al trabajar problemas en diferentes contextos, los profesores deben enfocarse en ayudar a los alumnos en las interpretaciones de los textos, así como a usar distintas representaciones semióticas, como la representación gráfica, algebraica, numérica y tabular. 
El profesor debe apoyar al estudiante para que tenga diferentes acercamientos en el proceso de solución; puede partir de lo intuitivo para llegar a lo formal. También es bienvenido el uso de diferentes recursos que le permitan al estudiante visualizar las situaciones planteadas, así como proponer discusiones que lo conduzcan a la reflexión sobre los elementos que contiene el problema y lo que se pretende obtener. Por ello, resulta fundamental estudiar los procesos de razonamiento que emplean los estudiantes, las habilidades que se pueden desarrollar, así como los procedimientos que pueden emplear.

\section{Referencias}

Aplications Android en Google Play. (2017). Statistics Quick Reference. Recuperado de https://play.google.com/store/apps/details?id=com.nuzedd.StatisticsQuickReference $\&$ hl=es_419.

Aplications Android en Google Play. (2017). Statics Calculator. Recuperado de https://play.google.com/store/apps/details?id=an.StatisticTools.

Batanero, C. y Sánchez, E. (2005). What is the nature of high school student's conceptions and misconceptions about probability? En G. A. Jones (Ed.), Exploring Probability in School: Challenges for Teaching and Learning (pp. 241-266). Nueva York, Estados Unidos: Springer.

Cai, J. (2003). What research tells us about teaching mathematics through problem solving? En. F. Lester (Ed.) Research and issues in teaching mathematics through problem solving (pp. 240-254). Reston, Estados Unidos: National Council of Teachers of Mathematics.

Duval, R. (2004). ¿Cómo describir para explicar? La(s) práctica(s) del lenguaje que la enseñanza de las ciencias y de las matemáticas exige y contribuye a desarrollar. En Ministerio de educación (Ed.), Los lenguajes de las ciencias (pp. 37-88). Madrid, España: Secretaría Técnica General.

D’Amore, B. (2006). Didáctica de las Matemáticas. Bogotá, Colombia: Cooperativa Editorial Magisterio.

Educatina. (2017). Recuperado de https://www.educatina.com/. 
Escuela Superior de Cómputo [ESCOM-IPN]. (2009). Programa de Estudios de Probabilidad y Estadística. Ciudad de México, México: ESCOM-IPN.

Filio, E. (2005). El lenguaje y el aprendizaje de las matemáticas. Un estudio desde la teoría de Chomsky. (Tesis de doctorado inédita). Centro de Investigación y Estudios Avanzados del Instituto Politécnico Nacional, Ciudad de México, México.

García, C. F. (2014). Lenguaje y comunicación en las matemáticas. Una aproximación teórica desde las matemáticas a los conceptos de lenguaje y comunicación en relación con los procesos de enseñanza y aprendizaje. (Tesis de maestría inédita). Universidad Nacional de Colombia, Medellín, Colombia.

Guevara, A. (2007). Lengua y significación de la realidad. En H. P. Reeder (Ed.), Lenguaje Dimensión lingüística y extralingüística del sentido (pp. 331-340). Bogotá, Colombia: Universidad Pedagógica Nacional.

Khan Academy. (2017). Recuperado de https://es.khanacademy.org/.

Lesh, R. y Zawojewski, J. (2007). Problem solving and modeling. En F. Lester (Ed.), Second Handbook of research on mathematics teaching and learning (pp. 763-802). Reston, Estados Unidos: National Council Teachers of Mathematics. Information Age Publishing.

Lester, F. y Charles, R. (Eds.). (2003) Teaching mathematics through problem solving: PreK-grade 6. Reston, Estados Unidos: National Council Teachers of Mathematics. Information Age Publishing.

Matthew Bognar. Google Store (2017). Probability Distributions @. Aplicación para celular. Recuperado de https://play.google.com/store/apps/developer?id=Matthew+Bognar\&hl.

Meyer P. (1999). Probabilidad y Aplicaciones Estadísticas. (1a ed.). Ciudad de México, México: Alhambra.

Monroe, E. E. (1996). Language and Mathematics: A Natural Connection for Achieving Literacy. Reading Horizons, 36 (5), 368-379.

Prados, M. y Cubero, R. (2005). Construcción del conocimiento y discurso educativo. Una aproximación al discurso de profesores y alumnos en la universidad. Avances en Psicología Latinoamericana, 23, 141-153. 
Polya, G. (1985), How to solve it. (2a ed.). New Jersey, Estados Unidos: Princeton University Press.

Pons, R. y Serrano, J. (2011). La adquisición del conocimiento: Una perspectiva cognitiva en el dominio de las matemáticas. Educación Siglo XXXI, 29(2), 117-138.

Punset, E. (2011). Excusas para no pensar. Cómo nos enfrentamos a las incertidumbres de nuestra vida. Barcelona, España: Ediciones Destino.

Rico, L. (1998). Errores y dificultades en el aprendizaje de las matemáticas. En. J. Kilpatrick., P. Gómez y L. Rico. (Eds.), Educación Matemática. Errores y dificultades de los estudiantes, resolución de problemas, evaluación e Historia (pp.69-104). Bogotá, Colombia: Universidad de los Andes.

Rojano, T. (1994). La matemática escolar como lenguaje. Nuevas perspectivas de investigación y enseñanza. Departamento de matemática educativa del CinvestavIPN, 12(1), 45-56.

Schoenfeld, A. H. (1992). Learning to think mathematically: problem solving, metacognition, and sense making in mathematics. En. D. Grouws (Ed.), Handbook of research on mathematics teaching and learning. A Project of the National Council of Teachers of Mathematics (pp. 334-370). Nueva York, Estados Unidos: Macmillan.

Sierpinska, A. y Lerman, S. (1996). Epistemologies of mathematics and of mathematics education. Bishop et al. (Eds.). International handbook of Mathematics Education, (pp. 827-876). Nueva York, Estados Unidos: Kluwer Academic Publishers.

Walpole, R., Myers, R., Myers, S. y Ye, K. (2012). Probabilidad y estadística para Ingeniería y Ciencias. Ciudad de México, México: Pearson. 


\begin{abstract}
Anexo 1
(Hoja que se entregó a los estudiantes para resolver los problemas de distribuciones de probabilidad).
\end{abstract}

Lea con atención y use la herramienta y/o fórmula conveniente para resolver los siguientes problemas. Todo problema debe tener procedimiento de solución.

1. Un escritor de libros comete, en promedio, 2 errores de procesamiento de texto por página en el primer borrador de su libro. ¿Cuál es la probabilidad de que en la siguiente página cometa:

a) ¿Cuatro o más errores?

b) ¿Ningún error?

2. Se estima que el número promedio de ratas de campo por acre, en un campo de 5 acres de trigo, es de 12. Calcule la probabilidad de que se encuentren menos de 7 ratas de campo.

a) en 1 acre dado;

b) en 2 de los siguientes 3 acres que se inspeccionen.

3. Suponga que la probabilidad de que una determinada persona crea un rumor acerca de las transgresiones de cierta actriz famosa es de 0.8. ¿Cuál es la probabilidad de que...

a) la sexta persona que escuche este rumor sea la cuarta en creerlo?

b) la tercera persona que escuche este rumor sea la primera en creerlo?

4.- Se estima que 4000 de los 10,000 residentes con derecho al voto de una ciudad están en contra de un nuevo impuesto sobre las ventas. Si se seleccionan al azar 15 votantes y se les pide su opinión,

a) ¿cuál es la probabilidad de que a lo sumo 7 estén a favor del nuevo impuesto?

b) ¿cuál es la probabilidad de que al menos 7 estén a favor del nuevo impuesto? 
5. Un empleado va a su oficina en el centro de la ciudad. El tiempo promedio para un viaje solo de ida es de 24 minutos, con una desviación estándar de 3.8 minutos. Si se supone que la distribución de los tiempos de viaje está distribuida normalmente.

a) ¿Cuál es la probabilidad de que un viaje tome al menos $1 / 2$ hora?

b) Calcule la duración mayor en la que se encuentra el 15\% de los viajes más lentos. 